\title{
De los principios de la bioética clínica a una bioética social para Chile
}

\author{
Francisco Javier León Correaa.
}

\section{From clinical to social bioethics in Chile}

The scope of clinical bioethics must be broadened to a social bioethics that tackles institutional and public health ethical problems and those related to the health system reform. The superficial application of the four bioethical principles is not enough to face these problems and assure a complete respect of individual rights. Using a qualitative research in bioethics, we will be able to deal with those problems that rise from health care in Chile and propose solutions to avoid inequities, to promote a humane care of patients, develop an institutional ethics and provide an efficient protection to people's rights. We propose to incorporate these social issues to the academic bioethical discussion and to develop a bioethics with complementary principles that can be used to solve cases and in decision making. We should use the means incorporated in our legislation such as Assistance Ethics Committees, Research Ethics Committees and the National Bioethics Commission, to pursue our objectives (Rev Méd Chile 2008; 136: 1078-82).

(Key words: Bioethics; Public health; Social medicine)

Recibido el 5 de noviembre, 2007. Aceptado el 22 de abril, 2008.

Centro de Bioética, Facultad de Medicina, Pontificia Universidad Católica de Chile. Santiago, Chile.

aDoctor en Filosofía, Magíster en Bioética

$E^{n}$ estos últimos años ha habido un desarrollo importante de la bioética en Chile. Necesitamos entender mejor los retos actuales que enfrenta y cuáles pueden ser las líneas que nos van a exigir una mayor dedicación en el futuro ${ }^{1}$, dentro de la situación general de América Latina ${ }^{2}$. Estamos en los comienzos de la difusión y aplicación de la bioética clínica, en el ámbito académico y en el clínico, con el desarrollo legislativo de los derechos y deberes de los usuarios del sistema de salud y la institucionalización de los Comités de

Correspondencia a: Dr. Francisco Javier León Correa. Centro Bioética. Edificio Gastroenterología, 5o piso. Alameda 340. Santiago. Fax: 6339785. E mail: fleonc@uc.cl
Ética en los hospitales. Pero no podemos quedarnos sólo en esto, es necesario ampliar la bioética clínica a la Atención Primaria de salud y, también desde los comités, desarrollar la ética institucional.

Objetivo de la actual acreditación de las instituciones de salud es vigilar el compromiso de respeto a los derechos de los pacientes ${ }^{3}$, y esto puede ser tarea de los Comités de Ética Asistencial en los hospitales, y de los comités de ética que puedan crearse en los Centros de Salud. Muchos de los problemas ético-clínicos, no provienen directamente de la relación profesional de la salud-paciente, sino de otros dos factores importantes: los problemas institucionales, y los problemas éticos planteados por las políticas y sistemas de salud. 
Para ello, es necesario dar el paso de una bioética centrada casi exclusivamente en los aspectos de ética clínica a una que hemos denominado social, porque debe afrontar tanto la promoción de la ética institucional, como el control desde la ética social de las políticas públicas de salud y de la evolución del propio sistema de salud, así como de las relaciones y adecuación entre el sistema y las exigencias y necesidades de la sociedad. Esto se puede lograr dando contenido a los principios de la bioética y buscando una fundamentación. No basta con una aplicación superficial de los cuatro principios clásicos, para asegurar en nuestros países el respeto a los derechos o la mejora de la situación vulnerable de gran parte de la población. Esta puede ser una de las herramientas para que desde los Comités de Ética Asistencial y Científica, y desde la Comisión Nacional de Bioética, ahora en constitución en nuestro país, se aborden también los aspectos de ética institucional y social

\section{LA DIFUSIÓN DE LA BIOÉTICA SOCIAL}

El propio desarrollo de la bioética global de Potter $^{4}$, el desarrollo de la ética institucional y empresarial aplicada a las instituciones de salud ${ }^{5}$, las propuestas de las éticas del desarrollo ${ }^{6-9} \mathrm{y}$ de la interculturalidad ${ }^{10}$, y la necesidad de legislar, han llevado a un reciente desarrollo de la bioética como una ética institucional, social y política ${ }^{1,11}$, con estudios también en Latinoamérica sobre justicia e igualdad en salud ${ }^{12}$, ética de las instituciones de salud ${ }^{5,13}$, género y ética en salud ${ }^{14}$, que van más allá de la bioética general y clínica.

Es un campo importante de promoción de los derechos humanos fundamentales -especialmente el derecho a la vida y a la salud- y de análisis de las consecuencias prácticas de la justicia en nuestras sociedades. Muchos problemas en ética clínica no hacen referencia exclusivamente a la relación médico-paciente, sino a defectos institucionales que están en la base de muchas quejas de los usuarios de nuestros hospitales ${ }^{15}$.

Es necesario pasar de una ética clínica a una bioética social, donde analicemos también la ética de las políticas de salud, de la gerencia de las instituciones de salud, de la distribución de recursos, de las condiciones laborales de los profesionales de la salud, etc. Pero la intención va más allá aún, y es ayudar al desarrollo de un debate plural sobre el papel de la ética en las sociedades democráticas.

\section{Propuesta de desarrollo de la bioÉtica como INSTRUMENTO DEL DEBATE ÉTICO-SOCIAL Y POĹTICO}

El éxito de la Bioética clínica ha sido la elaboración de una buena y eficaz metodología, la implementación de una buena propuesta de formación de los profesionales, y la penetración en el ámbito de la salud a través de los comités de ética de los hospitales. Para elaborar una Bioética social, debemos completar esa metodología y ampliar las funciones de los comités de ética, y por supuesto, es primordial la educación en el diálogo social, académico y profesional.

Adela Cortina -desde la ética dialógica- fundamenta la noción de persona como "interlocutor válido", aplicado específicamente a la Bioética ${ }^{16}$, y de ahí podemos señalar que:

- El debate en bioética debe ser plural, tolerante, con diálogos racionales.

- Deberemos establecer un consenso previo al debate, en torno al contenido de los derechos humanos fundamentales y los valores éticos de nuestra sociedad, y los valores que debe aportar la justicia social en el ámbito de la salud, así como jerarquizar los principios éticos en discusión ${ }^{17}$.

- Es necesario desde la bioética realizar un análisis detenido de los problemas éticos que necesariamente suscitan las reformas de la salud que se están implementando y por último.

- Es fundamental desarrollar la bioética en el ámbito de la salud pública y los sistemas de salud $^{18,19}$.

Debemos estudiar y proponer un nuevo modelo de relación médico-paciente. No cabe una aceptación acrítica de modelos externos, y tampoco una especie de indiferencia 0 abstención ante la evolución rápida desde un paternalismo a formas de un contractualismo más o menos individualista, o esquemas neoliberales poco concordes con la experiencia clínica.

Finalmente, debemos jerarquizar los principios bioéticos, sin reducciones fáciles y superficiales. Y 
también quizás debemos profundizar en las exigencias que aporta al análisis bioético el principio ético -no sólo jurídico- de justicia, y los cambios de perspectiva que nos aporta también la consideración del principio de beneficencia no sólo como la complementación del de no maleficencia, sino como deber responsable de solidaridad, más allá de la calidad o excelencia de los cuidados del profesional de la salud.

\section{Propuesta de desarrolo del modelo de los PRINCIPIOS EN UNA BIOÉTICA SOCIAL}

Ricardo Maliandi ha propuesto recientemente desde Argentina un desarrollo de los principios éticos en el área de la biotecnología, donde la no maleficencia y beneficencia serían el principio de precaución en la exploración genética; el de justicia, la no discriminación genética; y la autonomía, el principio de respeto por la diversidad genética ${ }^{20}$. Pienso que es necesario desarrollar los principios secundarios o intermedios que necesitamos para delimitar y concretar en la práctica los grandes principios generales. ¿Qué es la justicia en un caso ético-clínico concreto? Puede ser respeto a un derecho, puede ser protección de un paciente vulnerable, puede ser no discriminar de modo injusto los recursos en una decisión de limitación de tratamientos.

3.1 Principios intermedios de bioética clínica y social. La no maleficencia puede concretarse en tres principios: no abandono del paciente o sujeto de investigación; principio de precaución, que nos ayuda a evitar cualquier mala praxis; y principio de responsabilidad ante las consecuencias de las decisiones ético-clínicas, o de toma de medidas en salud pública.

Pero también existe el abandono, no sólo por parte del equipo profesional de salud, sino de la familia y la comunidad. Están, por tanto, los deberes de: responsabilidad familiar (no abandono familiar del paciente), y responsabilidad comunitaria (no abandono institucional y social).

El de justicia contiene otros varios: principio de equidad, en el acceso a los recursos de la salud; principio del respeto a los derechos o a la legalidad vigente; y más allá, el principio de protección, para conseguir efectivamente un nivel adecuado de justicia con los más vulnerables en la ética clínica o en la investigación biomédica ${ }^{21}$.

En cuanto a la autonomía, no es solamente el respeto por las decisiones libres, voluntarias e informadas del paciente, a través del proceso del consentimiento informado. Existe también más allá el deber ético de los profesionales de: promover la competencia efectiva del paciente, en la medida de lo clínicamente posible; y ayudar -sin paternalismos- a que el paciente pueda ejercer una libertad responsable.

Y más allá de la subrogación de la autonomía desde el punto de vista legal -muchas veces confundido con el ético- en los casos de falta de competencia momentánea o definitiva, está buscar al representante mejor de los intereses del paciente, y el papel de la familia en nuestra cultura. No se trata de una autonomía individualista, en muchos casos, sino del individuo en el ámbito familiar, y a veces comunitario, más amplio. Coexisten en nuestros países de hecho dos modelos, el de la salud privada y el de la salud pública. Pero no podemos mantener la idea de que la privada sería el ámbito del paciente que desea ser autónomo, mientras la pública queda -de hecho, no en los teóricos derechos- como el ámbito de la beneficencia y la justicia. Este planteamiento sería radicalmente injusto.

También corresponde al principio de autonomía: la participación de todos los ciudadanos en el control social y en la elaboración de las políticas de salud públicas; o en las líneas de investigación biomédica.

En la medida que todos sean más autónomos de hecho, deberán también participar en mayor grado en la delimitación de los valores éticos presentes en el sistema de salud y en las políticas de salud.

Por último, el principio de beneficencia, que es hoy en día mucho más que hacer al paciente el mayor bien posible según su propia escala de valores $^{22}$. Se entiende que el paciente: desea una atención de calidad y excelencia, y éstos son valores que deben incorporar los equipos y profesionales de la salud; requiere una medicina mucho más cercana, con una relación más personal, más humanizada.

El médico está ligado al paciente por un contrato profesional, y en el marco de una institución. Es, por tanto, el médico un agente que 
interviene en el nivel de la intención ética, el de la justicia. Pero a la vez, debe establecer relaciones personalizadas con los pacientes que atiende, y por eso su actuación se mueve en el nivel ético del "con y para los otros"23. No puede ignorar la justicia, pero debe actuar con beneficencia ${ }^{24}$. Y aquí son básicos los postulados de la ética del cuidado, que acerca la decisión al paciente concreto y singular, y no sólo al universalismo racional de los principios.

Y también es imprescindible el principio de solidaridad, más allá de la justicia, tanto a nivel social como individual. Una sociedad puede implementar un sistema que generalice la asistencia en salud a todos, con prestaciones de alta calidad, pero podría ser calificado como deshumanizado y distante por parte de los usuarios, y puede dar lugar a un aumento de las quejas o a una mala percepción de la atención recibida. Mientras que por el contrario, un sistema con menos recursos -económicos y humanos- puede ver de hecho compensadas sus deficiencias actuales por una solidaridad beneficente desde la propia sociedad o los individuos, pero también desde todas las instituciones públicas.

3.2 El desarrollo en la fundamentación de los principios. En cuanto a la fundamentación de los principios bioéticos, solemos poner el acento en las diferencias entre las diversas corrientes filosóficas o de escuela. Sí existen bastantes diferencias de enfoque entre los postulados de una ética utilitarista o una ética de consenso relativista, pero cabe un amplio diálogo entre otras corrientes que fundamentan las obligaciones éticas universales.

La base principal es una ética dialógica en la que todos somos "interlocutores válidos", que permite asentar las reglas éticas de los debates valóricos en nuestra sociedad democrática. No queremos seguir con un modelo paternalista de atención de salud, y debemos profundizar desde el diálogo y también desde las aportaciones del comunitarismo y el personalismo en los aspectos sociales que fundamentan la ética pública. Pensamos que no basta con algunas propuestas superficiales de teorías de liberación o de intervención, que abandonan el ámbito académico para entrar en el político.

La fundamentación de una ética neokantiana, deontológica, centrada en los deberes, es la base apropiada para los principios de no maleficencia y de justicia. El principio de autonomía puede sustentarse en dos visiones complementarias, la aportada por la ética dialógica y la aportada por el comunitarismo: somos "interlocutores válidos" de los demás, de todos los demás hombres racionales, pero insertos en una comunidad social y cultural, con valores propios. En cuanto a la beneficencia y solidaridad, la fundamentación más completa puede venir desde el personalismo, y de su primer principio de la "afirmación de la persona por sí misma".

No podemos restringir el objetivo de la bioética a la resolución de conflictos y dilemas en el enfrentamiento excepcional entre alguno o algunos de los cuatro principios. Es un papel importante, como vemos en la actuación de los Comités de Ética Asistencial, pero no es el único. En lo cotidiano, debemos aspirar a maximizarlos todos de modo armónico, en lo que se ha llamado el "principio de convergencia". Y este principio, exige tener en cuenta los aspectos institucionales y sociales de la ética.

\section{Implementación a tRaVÉs de los Centros de BioÉtica y COMItés DE ÉticA}

Para implementar esto, habría que incorporar al debate académico en los Centros de Bioética estos aspectos de su dimensión social. También habría que desarrollar el sistema de principios, con principios complementarios que puedan ser explicitados en las resoluciones de casos y toma de decisiones, de forma que en las decisiones de los Comités de Ética se incorporen de un modo más explícito los aspectos institucionales y sociales.

En tercer lugar, podemos utilizar los medios que ya están iniciados en nuestra legislación, y forman parte de los objetivos propuestos para los Comités de Ética Asistencial, los Comités de Ética Científica, los Comités de Ética Médica de los Servicios de Salud, y la Comisión Nacional de Bioética. Y habría que añadir, la creación de Comités de Ética a nivel de la Atención Primaria. En todos habría que incorporar a los futuros reglamentos esta función de velar por la ética institucional, cada uno en su nivel, y colaborar al debate de las políticas de salud y aspectos sociales y políticos del sistema de salud. 
Los Comités de Ética Asistencial "deben facilitar el adecuado cumplimiento de las funciones del hospi$\mathrm{tal}^{\prime 25}$, y deberían tener reglamentada su función de proponer a la institución protocolos y sugerencias de carácter institucional preventivo, para contribuir a la protección de los derechos de los pacientes 26,27, y asimismo los Comités de Ética Médica de los Servicios de Salud, que deben recibir y analizar la información de los dilemas éticos estudiados en los hospitales.

\section{REFERENCIAS}

1. León FJ. Por una Bioética social para Latinoamérica. Agora Philosophica, Mar del Plata 2005; 11: 19-26.

2. León FJ. Diez años de Bioética en América Latina: historia reciente y retos actuales. En: Lolas F ed. Diálogo y cooperación en salud. Diez años de Bioética en la OPS. Santiago: OPS-OMS, 2004; 145-52.

3. Estándares generales para prestadores institucionales de atención abierta. Respeto a la dignidad del paciente (DP). Santiago de Chile: Ministerio de Salud, 2007.

4. Acosta JR. Bioética para la sustentabilidad. La Habana, Centro Félix Varela, 2002.

5. Simon P. Ed. Ética de las organizaciones sanitarias. Nuevos modelos de calidad. Madrid: Triacastela, 2005.

6. Martín Navarro E. Ética para el desarrollo de los pueblos. Madrid: Trotta, 2000.

7. Goulet D. Ética del Desarrollo. Guía teórica y práctica. Madrid: Editorial IEPALA, 1999.

8. Cortina A. Ética para el desarrollo: un camino hacia la paz. Sistema, Madrid 2006; 192: 3-18.

9. ConIL J. Horizontes de economía ética. Aristóteles, Adam Smith, Amartya Sen. Madrid: Tecnos, 2006

10. SALAS R. Ética intercultural. Ensayos de una ética discursiva para contextos culturales conflictivos. Santiago: Ediciones UCSH, 2003

11. Kоттоw M. Salud pública, genética y ética. Revista de Saúde Pública, Sao Paulo, 2002; 36: 537-44.

12. Parenti F. Bioética y biopolítica desde América Latina. En: Acosta JR. Op.cit.: 171-84.

13. Cortina A. Ética de las instituciones de salud. Conferencia 8 mayo 2003. Santiago: Universidad del Desarrollo, 2003.

14. Género y Bioética. Número monográfico. Acta Bioethica, Santiago 2006, 12 (2).
Finalmente, la Comisión Nacional de Bioética tiene por ley la función de "asesorar a los distintos Poderes del Estado en los asuntos éticos que se presenten como producto de los avances científicos y tecnológicos en biomedicina, así como en las materias relacionadas con la investigación científica biomédica en seres humanos" ${ }^{28}$, y también debería por reglamento impulsar el debate bioético social y político.

15. Superintendencia de Salud. Estudio de Satisfacción de Usuarios. Santiago, septiembre 2007.

16. Cortina A. Ética aplicada y democracia radical. Madrid: Tecnos; 1993; 236-40.

17. Gracia D. Prólogo. En: Beaucham T, Childress JF. Principios de ética biomédica. Barcelona: Masson, 1999: 9-15.

18. Lolas F. La Bioética y los sistemas sanitarios en América Latina y el Caribe, en: III Congreso Nacional, Latinoamericano y del Caribe de Bioética. México DF: Comisión Nacional de Bioética, 1999.

19. Kotтow M. Bioética en Salud Pública. Santiago: Puerto de Palos, 2005.

20. MALANDI R. Ética: dilemas y convergencias. Buenos Aires: Biblos, 2006; 95-128.

21. Roland F, Kottow M. Principios bioéticos en Salud Pública: limitaciones y propuestas. Cad Saude Pública, Río de Janeiro: 2001; 17.

22. TAuber A. Patient Autonomy and the Ethics of Responsibility. Massachusetts: MIT Press, 2005.

23. Ricoeur P. Soi Même Comme un Autre. París: Editions du Seuil, 1990.

24. LEón FJ. Autonomía y beneficencia en ética clínica. Montevideo: Biomedicina 2006; 3: 257-60.

25. Ministerio de Salud. NGT no 2 Comités de Ética de los Servicios de Salud. Resolución exenta no 143, 1994. Modificaciones: no 1856, 1999.

26. Couceiro A, BECA JP. Los Comités de Ética Asistencial y las repercusiones jurídicas de sus informes. Rev Méd Chile 2006; 134: 517-9.

27. BeсA JP, Kotтow M. Orientaciones para Comités de Ética Hospitalaria. Santiago: Programa Regional de Bioética OPS/OMS, 1996.

28. Ministerio de Salud. Ley № 20.120 Sobre investigación científica en el ser humano. Diario Oficial № 38.570, 22-9-06. Artículos 15 y 16. Créase una Comisión Nacional de Bioética. 